\title{
Early detection of prostate cancer
}

\author{
Departamento de Cirurgia da Faculdade \\ de Medicina da USP
}

1. Why prostate cancer screening should be performed.

Prostate cancer was, until recently, less frequent than lung and colon neoplasms. It became the most frequent cancer in males from 1990 on (1). It is estimated that $8-10 \%$ of the male population will develop prostate cancer and $3 \%$ will die from it. It is a phenomenon of obvious clinical relevance (2). Furthermore, the rate of occurrence of this neoplasm has been increasing in the last years for reasons which are still unaccounted for. There are estimates indicating that between year 1980 and 2000, the number of prostate cancer cases and consequently deaths from it will grow in the $90 \%$ range (3). If one considers the annual expenses for the diagnosis and treatment of prostate cancer cases in the United States of America, this sum is in the U\$ 12 billions range (4). Having mentioned that, it is obvious the medical, socio-economic importance of prostate cancer and the interest it rises in the layperson and medical community.

The purpose for the introduction of prostate programs for the early detection of prostate cancer is grounded on the fact that only in the early stages of the disease, it can be successfully cured and that during this same early stages of the disease, there are clinical manifestations. The use of radical surgery or radiotherapy achieve a 50 to $85 \%$ cure rate in the cases of localized neoplasm whereas the anti-androgen therapy, usually employed for the patients with more advanced disease, slows down the disease progression without eliminating the neoplasm (1). Because only 30 to $50 \%$ of the prostate cancer cases are identified in the early stages of the disease, this early detection through screening programs represents the only way to improve the prognosis of the prostate cancer patients.

2. Patient selection for the screening programs.

The prostate cancer is very uncommon before age 50 unless when there is a family history for the disease or for breast cancer (5). Moreover, in many cases the neoplasm shows an indolent progression remaining in a quiescent state without producing clinical manifestations or death of the patient (1). This accounts for the discrepancy between the materialization of prostate cancer in autopsy studies and its clinical manifestations. For instance, in 80 year-old males, the rate of occurrence of prostate cancer in autopsy studies and the incidence of its clinical manifestations are respectively $44 \%$ and $28 \%$ (1). The systematic investigation for prostate cancer in advanced aged subjects is controversial because in a large number of these patients, the identification and treatment of the disease are not necessary (4).

In summary, the group of patients who benefit from an early prostate cancer diagnosis are those subjects aged 50 to 70 with a life prognosis of at least 10 years (2).

3 . Methods for prostate cancer early detection.

Prostate cancer can be diagnosed in its early stages by means of anal digital touch examination, measurement of the specific prostatic antigen and by means of an trans-rectal ultrasound $(1,2,4,6,7,8)$

Digital Rectal Examination (DRE) when performed by an experienced examiner, allows the detection of masses about 0.5 centimeter in diameter. This is useful in terms of the clinical point of view. Studies by Mc Neal et al have demonstrated that the potentially aggressive tumors have always at least one cubic centimeter in volume $(9,10)$ and a mass of this size is easily detectable during a DRE. However, this method fails in neoplasms which are originated in the transitional in the transitional zone (periurethral). These tumors account for nearly $25 \%$ of all cases of prostate cancer.

Measurement of the specific prostatic antigen in the serum (SPA) represent another alternative in the prostate cancer detection. The addition of the SPA to the DRE enhances in $50 \%$ the chances of diagnosing prostate cancer (11). Additionally, when one considers the PSA alone in levels above to $4.0 \mathrm{ng} / \mathrm{ml}$ (Tandem-R PSA, Hybritech) the presence of pros- 
tate cancer is confirmed in nearly $25 \%$ of the cases. Several of these cases display unremarkable PDT and ultrasound (6).Nonetheless, the employment of PSA alone is insufficient for the clinical detection of early prostate cancer because 21 to $43 \%$ of the patients with definitely diagnosed prostate cancer show a normal serum level of this marker (PSA) (2).The prostate investigation by means of an trans-rectal ultrasound study (TRUS) represent another method to diagnose local neoplasms. TRUS usually demonstrates an increased echo in comparison with the healthy prostate parenchyma (12). TRUS allows the detection of masses of $7 \mathrm{~mm}$ in diameter and up. This renders the TRUS abnormal in $85 \%$ of the cases with prostate cancer (2). The inconvenience of TRUS is the occurrence of false positives which renders its specificity lower and therefore its predictive value. This is observed in nearly $2 / 3$ of the patients and it is due to the presence of infarcts, prostatis, hyperplasic nodules, or prostate cysts (12).In the table depicted below, the sensibility, specificity indexes for the PDT, PSA and TRUS in the diagnosis of prostate cancer are listed. As shown in the table, the employment of each of these methods alone allow detection of a smaller percentage of the cases. Therefore, taking into consideration, the costs and benefits of each method, some guidelines for the early prostate cancer have been established. Initially, a PDT combined with PSA. The TRUS is employed only of both PDT and PSA are abnormal $(1,2,5)$.

\begin{tabular}{lrrr}
\hline \hline Methods & Sensibility & Specificity & $\begin{array}{r}\text { Positive } \\
\text { predictive value }\end{array}$ \\
\hline PDT & $69-89 \%$ & $84-98 \%$ & $26-35 \%$ \\
Serum PSA & $57-79 \%$ & $56-68 \%$ & $40-49 \%$ \\
TRUS & $36-85 \%$ & $41-79 \%$ & $27-36 \%$ \\
\hline
\end{tabular}

